Homology, Homotopy and Applications, vol.6(1), 2004, pp.33-47

\title{
ON DIMENSIONS IN BREDON HOMOLOGY
}

\author{
BRITA E. A. NUCINKIS \\ (communicated by Johannes Huebschmann)
}

\begin{abstract}
We define a homological and cohomological dimension of groups in the context of Bredon homology and compare the two quantities. We apply this to describe the Bredon-homological dimension of nilpotent groups in terms of the Hirsch-rank. In particular this implies that for virtually torsion-free nilpotent groups the Bredon cohomological dimension is equal to the virtual cohomological dimension.
\end{abstract}

\section{Introduction}

The definition of Bredon cohomology groups for $G$-CW-complexes for finite groups $G$ goes back to Bredon [3] and was further investigated by Slominska, see e.g. $[\mathbf{2 2}]$. The definitions can easily be extended to infinite groups $G$ and have recently regained attention through their use in equivariant obstruction theory [17] and through their connection to group actions on proper $G$-spaces $[\mathbf{1 3}, \mathbf{1 8}, \mathbf{2}, \mathbf{1 2}, \mathbf{1 9}]$. Bredon homology also features in the Baum-Connes conjecture; a nice exposition of how the two relate can be found in $[\mathbf{1 8}]$.

We say a $G$-CW-complex $X$ is an $\underline{E} G$, or universal proper $G$-space, if $X^{H}$ is contractible when $H \leqslant G$ is finite and $X^{H}$ is empty otherwise. The minimal dimension of such an $\underline{E} G$ is denoted by $\operatorname{gd} G$. By analogy with ordinary cohomology one defines the Bredon-cohomological dimension $\underline{\operatorname{cd}} G$ and the Bredon-homological dimension hd $G$ of a group. The augmented cellular chain complex of an $\underline{E} G$ gives a Bredon projective resolution for the group and hence $\operatorname{cd} G \leqslant \operatorname{gd} G$. A detailed introduction to Bredon homology follows in Section 2. As for the torsion-free case [8] it was shown by Lück [13, Theorem 13.19] that, provided $\underline{\mathrm{cd}} G \geqslant 3$, the two quantities are equal. If either dimension is one, it follows from a result of Dunwoody [7] that they are equal. In fact, it turns out [2] that there are examples of groups where $\underline{\operatorname{cd}} G=2$ yet $\underline{\operatorname{gd}} G=3$.

A question which has received some attention is how $\underline{\mathrm{cd}} G$ and $\underline{\operatorname{gd}} G$ behave under group-extensions. Originally these questions arose for virtually torsion-free groups without mentioning the spaces $\underline{E} G$ or the dimensions $\underline{\mathrm{cd}} G$ and $\operatorname{gd} G$ by the names used here. Let $H \leqslant G$ be a torsion-free subgroup of finite index $k$. Then by Serre's theorem, see [5, Theorems VIII.3.1, VIII.11.1], $\operatorname{gd} G \leqslant k(\mathrm{~g} d H)$. It was Brown who first asked whether it can be shown that $\underline{\operatorname{gd}} G=\mathrm{g} d H[\mathbf{5}, \mathbf{4}]$, a question which has

Received September 26, 2003, revised January 27, 2004; published on March 4, 2004. 2000 Mathematics Subject Classification: 20J05, 18G, 55N25, 20F18.

Key words and phrases: Bredon homology, (co)homological dimension, Hirsch length. (C) 2004, Brita E. A. Nucinkis. Permission to copy for private use granted. 
become known as the Brown conjecture. In [12] Ian Leary and the author gave examples where there is a strict inequality $\operatorname{gd} G>g d H$. Lück $[\mathbf{1 4}]$ extended Serre's result to arbitrary group extensions $H \hookrightarrow G \rightarrow Q$, where the group $Q$ has a bound $d$ on the orders of its finite subgroups: $\operatorname{gd} G \leqslant \operatorname{gd} H+d(\operatorname{gd} Q)$. Martinez [16] developed spectral sequences in Bredon homology and cohomology for various families $\mathfrak{F}$ of subgroups. Applying these to some special families of finite subgroups she obtained similar bounds for $\underline{\operatorname{cd}} G$ in terms of $\underline{\operatorname{cd} H}$ and $\underline{\operatorname{cd}} Q$.

The motivation for this work was to find classes of groups for which the Brown conjecture holds. It is known to hold for polycyclic groups, see [15, Example 5.25]. Here we show it for nilpotent groups using a purely algebraic approach following ideas of Stammbach [23] and Bieri [1] who related the (co)homological dimension of soluble groups to the Hirsch length $h G$. For torsion-free soluble groups a result of Stammbach [23] implies that hd $G=h G$, and, if in addition $G$ is countable, then $h G \leqslant \operatorname{cd} G \leqslant h G+1$. For a detailed account of these facts see [1]. After proving some preliminary results in Section 3, including an analogue of Lazard's result [11] for arbitrary rings, that flat modules are direct colimits of finitely generated free modules, we shall look at cohomological and homological dimensions of groups in the Bredon setting. The main result of Section 4 is that for arbitrary countable groups $G$,

$$
\underline{\mathrm{hd}} G \leqslant \underline{\mathrm{cd}} G \leqslant \underline{\mathrm{hd}} G+1 .
$$

Furthermore, for soluble groups, $h G \leqslant \underline{\mathrm{hd}} G$. In Section 5 we consider group extensions and give a short account of some of Martinez' results applied to families of finite subgroups. In Section 6 we apply these results to nilpotent groups. In particular, we show that for nilpotent groups G (Theorem 6.1),

$$
\underline{\mathrm{hd}} G=h G \text {. }
$$

and that for virtually torsion-free nilpotent groups (Theorem 6.3)

$$
\underline{\mathrm{cd}} G=v c d G,
$$

which also implies that the Brown conjecture holds for this class of groups.

\section{Preliminaries on Bredon modules}

We begin with a few basic definitions concerning Bredon-modules, most of which can be found in Lück's [13] or tom Dieck's [6] books. Another good reference for background material is Mislin's survey article [18].

Let $G$ be an arbitrary group and $\mathfrak{F}$ a family of subgroups closed under subgroups and conjugation. Even though our main applications will be for families $\mathfrak{F}$ of finite subgroups of $G$, the results of the next two sections hold in greater generality. We shall consider the orbit category $\mathcal{O}_{\mathfrak{F}} G$, which is a category with objects the cosets $G / H$, where $H \in \mathfrak{F}$ and the morphisms are $G$-maps $G / H \rightarrow G / K$, where $G / H$ and $G / K$ are objects in $\mathcal{O}_{\mathfrak{F}} G$. We now consider functor categories from $\mathcal{O}_{\mathfrak{F}} G$ to $\mathfrak{A} \mathfrak{b}$, the category of abelian groups. We denote by $\operatorname{Mod}_{\mathfrak{F}-} G$, the category of contravariant functors $M(-): \mathcal{O}_{\mathfrak{F}} G \rightarrow \mathfrak{A} \mathfrak{b}$ and by $G$-Mod $\operatorname{Mod}_{\mathfrak{F}}$ the category of covariant functors 
$N(-): \mathcal{O}_{\mathfrak{F}} G \rightarrow \mathfrak{A} \mathfrak{b}$. Since for $\mathfrak{F}=\{e\}$, the category $\operatorname{Mod}_{\mathfrak{F}} G$ is just the category of right $G$-modules and $G-\operatorname{Mod}_{\mathfrak{F}}$ is the category of left $G$-modules we shall usually call the objects of these categories right $\mathcal{O}_{\mathfrak{F}} G$-modules or left $\mathcal{O}_{\mathfrak{F}} G$-modules respectively. In case we are just mentioning $\mathcal{O}_{\mathfrak{F}} G$-modules we imply that the statement holds for both categories.

Many of the properties of $G$-modules can be more or less directly taken over to $\mathcal{O}_{\mathfrak{F}} G$-modules. For example, by taking things object-wise we have kernels and cokernels as well as arbitrary coproducts. A sequence $M^{\prime} \rightarrow M \rightarrow M^{\prime \prime}$ of $\mathcal{O}_{\mathfrak{F}} G$-modules is exact if and only if $M^{\prime}(G / H) \rightarrow M(G / H) \rightarrow M^{\prime \prime}(G / H)$ is exact for all $H \in \mathfrak{F}$. Also submodules, intersections and sums of modules are defined objectwise. We can also define chain complexes, exact sequences, resolutions and chain homotopies in the usual manner.

We shall now describe how to construct projectives in $\operatorname{Mod}_{\mathfrak{F}^{-}} G$. A nice thorough account can be found in [18]. We say a module $P$ in $\operatorname{Mod}_{\mathfrak{F}}-G$ is projective if the functor:

$$
\operatorname{mor}(P,-): \operatorname{Mod}_{\mathfrak{F}} G \rightarrow \mathfrak{A} \mathfrak{b}
$$

is exact. Let $K \in \mathfrak{F}$ and consider the right $\mathcal{O}_{\mathfrak{F}} G$-module $P_{K}$, which is defined as follows:

$$
P_{K}(G / H)=\mathbb{Z} \operatorname{mor}(G / H, G / K) .
$$

$\mathbb{Z} m o r(G / H, G / K)$ denotes the free abelian group on the set $\operatorname{mor}(G / H, G / K)$. An application of a Yoneda-type argument, see [18], yields

$$
\operatorname{mor}\left(P_{K}, M\right) \cong M(G / K),
$$

which in turn gives the desired exactness of $\operatorname{mor}\left(P_{K},-\right)$. Obviously, a coproduct of projectives is again projective and therefore we have, for an arbitray $M \in \operatorname{Mod}_{\mathfrak{F}}-G$ the following epimorphism of a projective onto $M$ :

$$
\coprod_{H \in \mathfrak{F}}\left(\coprod_{M(G / H)} P_{H}\right) \rightarrow M
$$

In particular every right $\mathcal{O}_{\mathfrak{F}} G$-module $M$ admits a projective resolution, denoted $P_{*} \rightarrow M$ which is used to define the derived functors $\operatorname{Ext}_{\mathfrak{F}}^{*}(M, N)$, which are contravariant in the first and covariant in the second variable:

$$
\operatorname{Ext}_{\mathfrak{F}}^{*}(M, N)=H^{*}\left(\operatorname{mor}\left(P_{*}, N\right)\right)
$$

There is also the obvious definition of the projective dimension, denoted $p d_{\mathfrak{F}} M$, of a right $\mathcal{O}_{\mathfrak{F}} G$-module $M$. It is the minimal length of a projective resolution of $M$.

We also use projective resolutions to define a $\operatorname{Tor}_{*}^{\mathfrak{F}}(M, N)$. To do this we introduce tensor products in our category. There are two such products which are of interest to us: the tensor product over $\mathbb{Z}$ and the tensor product over the Orbitcategory $\mathcal{O}_{\mathfrak{F}} G$. Let $M(-) \in \operatorname{Mod}_{\mathfrak{F}}-G$ and $N(-) \in G$-Mod $\operatorname{Mo}_{\mathfrak{F}}$. We define the tensor product over $\mathbb{Z}$ as the composite of the following two functors:

$$
M(-) \otimes N(-): \mathcal{O}_{\mathfrak{F}} G \times \mathcal{O}_{\mathfrak{F}} G \rightarrow \mathfrak{A b} \times \mathfrak{A} \mathfrak{b} \stackrel{\otimes \mathbb{Z}}{\longrightarrow} \mathfrak{A} \mathfrak{b} .
$$


The definition of a tensor product over $\mathcal{O}_{\mathfrak{F}} G$ needs a bit more work. One can define it explicitly or use a universal property as done by Yoneda. For a nice account concerning the universal property as well as other results on tensor products of functors see [9]. For definitions in our sense and some of the facts now to follow see [13] and [18]. Let $\varphi: G / K \rightarrow G / L$ be a $G$-map. We now define the categorical tensor product to be the abelian group

$$
M(-) \otimes_{\mathfrak{F}} N(-)=\bigoplus_{K \in \mathfrak{F}} M(G / K) \otimes N(G / K) / \sim
$$

where $\sim$ is the equivalence relation generated by $\varphi^{*}(m) \otimes n=m \otimes \varphi_{*}(n)$ with $m \in M(G / L)$ and $n \in N(G / K)$.

Let $\operatorname{Tor}_{n}^{\mathfrak{F}}(-, N)$ denote the $\mathrm{n}$-th left derived functor of the tensor product $-\otimes_{\mathfrak{F}} N$. We say a right $\mathcal{O}_{\mathfrak{F}} G$-module $M$ is flat if the functor $M \otimes_{\mathfrak{F}}$ - is exact. Since, by the Yoneda isomorphism,

$$
P_{K}(-) \otimes_{\mathfrak{F}} N(-) \cong N(G / K)
$$

for all $N \in G-\operatorname{Mod}_{\mathfrak{F}}$ and the tensor product commutes with coproducts, one can conclude that projective right $\mathcal{O}_{\mathfrak{F}} G$-modules are flat. Define the flat dimension $\left(f l d_{\mathfrak{F}}\right)$ of a right $\mathcal{O}_{\mathfrak{F}} G$-module as the minimal length of a flat resolution. Hence, for all $M \in \operatorname{Mod}_{\mathfrak{F}^{-}} G$ :

$$
f l d_{\mathfrak{F}} M \leqslant p d_{\mathfrak{F}} M
$$

To define free right $\mathcal{O}_{\mathfrak{F}} G$-modules we follow $[\mathbf{1 3}, 9.16,9.17]$. Firstly we have to say what we mean by an $\operatorname{Obj}\left\{\mathcal{O}_{\mathfrak{F}} G\right\}$-set $\Delta$, or $\mathfrak{F}$-set $\Delta$ for short. This is a family $\left\{\Delta_{K} \mid K \in \mathfrak{F}\right\}$ of sets $\Delta_{K}$. An $\mathfrak{F}$-map between two $\mathfrak{F}$-sets $\Delta$ and $\Omega$ is a family of maps $\left\{\Delta_{K} \rightarrow \Omega_{K} \mid K \in \mathfrak{F}\right\}$. There is an alternative way of thinking of $\mathfrak{F}$-sets, namely as pairs $(\Delta, \varphi)$ with $\varphi: \Delta \rightarrow \operatorname{Obj}\left(\mathcal{O}_{\mathfrak{F}} G\right)$, where $\Delta_{K}=\varphi^{-1}(G / K)$. By forgetting the structure we obtain an $\mathfrak{F}$-set from any $\mathcal{O}_{\mathfrak{F}} G$-module. We can now define the free $\mathcal{O}_{\mathfrak{F}} G$-module on an $\mathfrak{F}$-set as the left adjoint to the just mentioned forgetful functor $\operatorname{Mod}_{\mathfrak{F}}-G \rightarrow \mathfrak{F}$-set and we have the usual universal property: A right $\mathcal{O}_{\mathfrak{F}} G$-module $F$ is called free over the $\mathfrak{F}$-set $\Delta$ if $\Delta$ is a $\mathfrak{F}$-subset of $F$, i.e. $\Delta_{K} \subset F(G / K)$ for each $K \in \mathfrak{F}$ and if each $\mathfrak{F}$-map $f: \Delta \rightarrow N$ with $N$ an arbitrary right $\mathcal{O}_{\mathfrak{F}} G$-module has a unique extension by an $\mathcal{O}_{\mathfrak{F}} G$-module map $\tilde{f}: F \rightarrow N$.

As an example, the right modules $P_{K}$ defined above are free on the $\mathfrak{F}$-set $\Delta$ with $\Delta_{K}=\{G / K\}$, the set with one element $G / K$, and $\Delta_{H}=\varnothing$ for $H \neq K$. Also, the module $\coprod_{K \in \mathfrak{F}} P_{K}$ is free on the $\mathfrak{F}$-set $\Delta$ with $\Delta_{K}=\{G / K\}$ as above for all $K \in \mathfrak{F}$. In general, given an $\mathfrak{F}$-set $(\Delta, \varphi)$, we can construct the free module $F_{\Delta}$ as

$$
F_{\Delta}=\coprod_{\delta \in \Delta} P_{\varphi(\delta)} .
$$

A free right $\mathcal{O}_{\mathfrak{F}} G$-module is finitely generated if it is free on a finite $\mathfrak{F}$-set $\Delta$ or, equivalently, if it is a finite coproduct of free modules of the form $P_{K}$ for $K \in \mathfrak{F}$. Let $M$ be a right $\mathcal{O}_{\mathfrak{F}} G$-module and $S \subset M$ an $\mathfrak{F}$-subset. Then the submodule generated by $S$ is the smallest $\mathcal{O}_{\mathfrak{F}} G$-submodule containing $S$. Hence, a right $\mathcal{O}_{\mathfrak{F}} G$-module is finitely generated if and only if it is a quotient of a finitely generated free module. 
A right $\mathcal{O}_{\mathfrak{F}} G$-module $M$ is said to be finitely presented if there is a short exact sequence $N \hookrightarrow F \rightarrow M$ of $\mathcal{O}_{\mathfrak{F}} G$-modules with $F$ finitely generated free and $N$ finitely generated.

Later on we shall require $\mathcal{O}_{\mathfrak{F}} G$-modules which have similar properties to duals of finitely generated projective $\mathbb{Z} G$-modules. It turns out that in the Bredon setting we can't make as general a definition as for ordinary $G$-modules.

Let $P_{K}$ be the free right $\mathcal{O}_{\mathfrak{F}} G$-module of rank 1 as explained above. We define its dual

$$
P^{K}=\mathbb{Z} \operatorname{mor}(G / K,-),
$$

which is now a left $\mathcal{O}_{\mathfrak{F}} G$-module. For arbitrary finitely generated free right $\mathcal{O}_{\mathfrak{F}} G$ modules $F=\coprod_{K \in \mathfrak{F}^{\prime}} P_{K}\left(\mathfrak{F}^{\prime}\right.$ a finite subfamily of $\left.\mathfrak{F}\right)$ we set

$$
F^{*}=\coprod_{K \in \mathfrak{F}^{\prime}} P^{K}
$$

Analogous to the contravariant case $([\mathbf{1 8}])$ one can prove that

$$
\operatorname{mor}\left(P^{K}, N\right)=N(G / K)
$$

for every $N \in G$-Mod $\operatorname{Fon}_{\mathfrak{F}}$ and every $K \in \mathfrak{F}$. Hence, the $P^{K}$ and also $F^{*}$ are projective left $\mathcal{O}_{\mathfrak{F}} G$-modules and one can also show that every left $\mathcal{O}_{\mathfrak{F}} G$-module has a free mapping onto it.

An application of the above yields a one-to-one correspondence between $\operatorname{mor}\left(P_{K}, P_{L}\right)$ and $\operatorname{mor}\left(P^{L}, P^{K}\right)$, since

$$
\begin{aligned}
\operatorname{mor}\left(P_{K}, P_{L}\right) & =P_{L}(G / K)=\mathbb{Z} \operatorname{mor}(G / K, G / L) \\
& =P^{K}(G / L)=\operatorname{mor}\left(P^{L}, P^{K}\right)
\end{aligned}
$$

by the above. Hence, for abitrary finitely generated free $\mathcal{O}_{\mathfrak{F}} G$-modules $P$ and $Q$ we have a one-to-one correspondence

$$
\operatorname{mor}(P, Q)=\operatorname{mor}\left(Q^{*}, P^{*}\right) .
$$

It is worth mentioning that the whole construction works the other way round, too. For arbitrary finitely generated free left $\mathcal{O}_{\mathfrak{F}} G$ - modules $P$ we have a dual $P^{*}$, which is a finitely generated free right $\mathcal{O}_{\mathfrak{F}} G$-module and

$$
P^{* *}=P \text {. }
$$

in either setting.

Now let $M \in \operatorname{Mod}_{\mathfrak{F}^{-}} G$ and $P^{K}=\mathbb{Z} \operatorname{mor}(G / K,-) \in G$-Mod $\operatorname{F}_{\mathfrak{F}}$. We define a map

$$
\Psi: M(G / K) \rightarrow M(-) \otimes_{\mathfrak{F}} P^{K}(-)
$$

by $m \mapsto m \otimes i d \in M(G / K) \otimes P^{K}(G / K)$. This map is obviously injective. Take an arbitrary basis-element $m \otimes \varphi \in M(G / H) \otimes P^{K}(G / H)$, where $\varphi: G / K \rightarrow G / H$. This $\varphi$ can be viewed as the composite $\varphi \circ i d$, hence $m \otimes \varphi(i d)=\varphi^{*}(m) \otimes i d \in$ $M(G / K) \otimes P^{K}(G / K)$ and hence $\Psi$ is onto.

Applying this and the fact that the tensor-product and mor commute with finite coproducts, we obtain the following isomorphism

$$
M \otimes_{\mathfrak{F}} P^{*} \cong \operatorname{mor}(P, M)
$$


for finitely generated free $P \in \operatorname{Mod}_{\mathfrak{F}^{-}} G$ and arbitrary $M \in \operatorname{Mod}_{\mathfrak{F}^{-}} G$.

\section{Flat Bredon modules}

To begin this section we collect a few facts about colimits in general and also in the category $\operatorname{Mod}_{\mathfrak{F}^{-}} G$. A general definition of a colimit can be found in Weibel's book [24, pp. 54-56]. Let $I$ be an indexing category and $\left(M_{i}\right)_{i \in I}$ a family of objects in $\operatorname{Mod}_{\mathfrak{F}^{-}} G$. The colimit $\operatorname{colim} M_{i}$ is a functor from $\operatorname{Mod}_{\mathfrak{F}^{-}} G^{I}$ to $\operatorname{Mod}_{\mathfrak{F}}-G$, which is left adjoint to the diagonal functor $\Delta: \operatorname{Mod}_{\mathfrak{F}^{-}} G \rightarrow \operatorname{Mod}_{\mathfrak{F}^{-}} G^{I}$. Hence colimits are right exact in $\operatorname{Mod}_{\mathfrak{F}^{-}} G$ if they exist. Recall that the coproduct for a family $\left(M_{i}\right)_{i \in I}$, of objects in $\operatorname{Mod}_{\mathfrak{F}^{-}} G$ is defined objectwise by $\left(\coprod M_{i}\right)(G / H)=\bigoplus\left(M_{i}(G / H)\right)$. By a standard result, see e.g. [24], Proposition 2.6.8, it follows that the colimit colim $M_{i}$ can be viewed as the cokernel of the map:

$$
\coprod_{\varphi: i \rightarrow j} M_{i} \rightarrow \coprod_{i \in I} M_{i}
$$

and therefore exists and is defined objectwise. Let $I$ be a filtered category. We denote the filtered colimit of a family $M_{i}$ by $\operatorname{colim} M_{i}$. Since filtered colimits are exact for modules over arbitrary rings, we have shown the following:

Lemma 3.1. Filtered colimits are exact in $\operatorname{Mod}_{\mathfrak{F}-} G$.

The main result of this section is the following Bredon analogue to Lazard's [11] theorem.

Theorem 3.2. The following conditions on a right $\mathcal{O}_{\mathfrak{F}} G$-module $M$ are equivalent.

(i) $M$ is flat.

(ii) For every finitely presented $\mathcal{O}_{\mathfrak{F}} G$-module $P$ and $\mathcal{O}_{\mathfrak{F}} G$-module homomorphisms $\varphi: P \rightarrow M$ there exists a finitely generated free $\mathcal{O}_{\mathfrak{F}} G$-module $F$ such that $\varphi$ factors through $F$.

(iii) $M$ is the filtered colimit of finitely generated free $\mathcal{O}_{\mathfrak{F}} G$-modules.

Proof. (i) $\Rightarrow$ (ii): Since $P$ is finitely presented there exists an exact sequence

$$
F_{1} \stackrel{f}{\longrightarrow} F_{0} \stackrel{g}{\longrightarrow} P \rightarrow 0
$$

with $F_{1}$ and $F_{0}$ both finitely generated free right $\mathcal{O}_{\mathfrak{F}} G$-modules. Taking duals we obtain an exact sequence of left $\mathcal{O}_{\mathfrak{F}} G$-modules

$$
F^{\prime} \stackrel{h}{\longrightarrow} F_{0}^{*} \stackrel{\hat{f}}{\longrightarrow} F_{1}^{*}
$$

where $F^{\prime} \in G-\operatorname{Mod}_{\mathfrak{F}}$ is free. Since $M$ is flat this yields an exact sequence

$$
M \otimes_{\mathfrak{F}} F^{\prime \prime} \stackrel{i d \otimes h}{\longrightarrow} M \otimes_{\mathfrak{F}} F_{0}^{*} \stackrel{i d \otimes \hat{f}}{\longrightarrow} M \otimes_{\mathfrak{F}} F_{1}^{*} .
$$

By (3) we have the following commutative diagram:

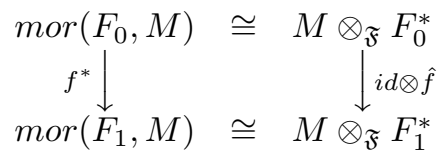


Since $\varphi \circ g \circ f=f^{*}(\varphi \circ g)=0$ and (6) is exact, there exists an element $x \in M \otimes_{\mathfrak{F}} F^{\prime}$ such that $(i d \otimes h)(x)=\varphi \circ g$. Now there exists a finitely generated free left $\mathcal{O}_{\mathfrak{F}} G$ submodule $F^{*}$ of $F^{\prime}$ such that

$$
x \in M \otimes_{\mathfrak{F}} F^{*} \hookrightarrow M \otimes_{\mathfrak{F}} F^{\prime}
$$

Denote by $\hat{k}$ the composite $F^{*} \hookrightarrow F^{\prime} \stackrel{h}{\longrightarrow} F_{0}^{*}$ and by $k$ its transpose $F_{0} \rightarrow F$, where $F=F^{* *}$.

Since (5) is exact it follows from (2) that $0=k \circ f \in \operatorname{mor}\left(F_{1}, F\right)$. Passing onto quotients we obtain a well defined map $\alpha \in \operatorname{mor}(P, F)$ such that $\alpha \circ g=k$. Since $F^{*}$ is a finitely generated free left $\mathcal{O}_{\mathfrak{F}} G$-module the isomorphism (3) yields that $x \in M \otimes_{\mathfrak{F}} F^{*}$ defines a map $\beta \in \operatorname{mor}(F, M)$. Another look at a diagram, similar to the one above yields

$$
\varphi \circ g=(i d \otimes \hat{k})(x)=k^{*}(\beta)=\beta \circ k .
$$

Passing onto quotients we obtain the desired equality $\beta \alpha=\varphi$.

(ii) $\Rightarrow$ (iii): Let $F_{X}$ denote the free right $\mathcal{O}_{\mathfrak{F}} G$-module on the $\mathfrak{F}$-set $X$. We can view the right $\mathcal{O}_{\mathfrak{F}} G$-module $M$ as an $\mathfrak{F}$-set $(M, \varphi)$ by forgetting the structure $(\varphi: M \rightarrow$ $\operatorname{Obj}\left(\mathcal{O}_{\mathfrak{F}} G\right)$ and $\varphi^{-1}(G / K)=M(G / K)$ with the module structure forgotten).

Take an arbitrary infinite countable $\mathfrak{F}$-set $(N, \psi)$ and form the direct product $(M, \varphi) \times(N, \psi)=M \times N$. Let $F_{M \times N}$ denote the free right $\mathcal{O}_{\mathfrak{F}} G$-module on $M \times N$, $\pi: F_{M \times N} \rightarrow M$ the canonical projection and $K=\operatorname{ker}(\pi)$.

Now define $E$ to be the category which has as objects pairs $(I, S)$, where $I$ is a finite $\mathfrak{F}$-subset of $M \times N$ and $S$ is a finitely generated $\mathcal{O}_{\mathfrak{F}} G$-submodule of $K$. There is a unique arrow $(I, S) \rightarrow\left(I^{\prime}, S^{\prime}\right)$ in $E$ if and only if $I$ is an $\mathfrak{F}$-subset of $I^{\prime}$ and $S$ is an $\mathcal{O}_{\mathfrak{F}} G$-submodule of $S^{\prime} . E$ is a filtered category as $(I, S) \in \operatorname{Obj}(E)$ and $\left(I^{\prime}, S^{\prime}\right) \in \operatorname{Obj}(E)$ both have an arrow to $\left(I \cup I^{\prime}, S+S^{\prime}\right) \in \operatorname{Obj}(E)$.

Consider $e=(I, S) \in E$ and the right $\mathcal{O}_{\mathfrak{F}} G$-module $M_{e}=F_{I} / S$. Since $S \subset K$, the canonical injection $F_{I} \hookrightarrow F$ induces a morphism $\Theta_{e}: M_{e} \rightarrow M$. Similarly one sees that there are induced morphisms $\varphi_{e^{\prime}}^{e}: M_{e} \rightarrow M_{e^{\prime}}$ for each arrow $e \rightarrow e^{\prime}$. Hence this construction gives us a functor $\Lambda: E \rightarrow \operatorname{Mod}_{\mathfrak{F}^{-}} G$, which sends $e \mapsto M_{e}$. By the universal property for filtered colimits we obtain a unique morphism

$$
\Theta: \underset{\operatorname{colim}}{\longrightarrow} M_{e} \rightarrow M \text {. }
$$

$\Theta$ is obviously surjective: for each $m \in M$ there is the element $e=(\{m\}, 0)$, where $\{m\}$ is viewed as an $\mathfrak{F}$-set.

To show that $\Theta$ is injective we use the explicit description of colimits (4). Every element $y$ in $\underset{\longrightarrow}{\operatorname{colim}} M_{e}$ is the image of some $y_{e} \in M_{e}(e=(I, S))$. In case $y \in \operatorname{ker}(\Theta)$, it follows that $\Theta_{e}\left(y_{e}\right)=0$. We show that there is an $e^{\prime} \in E$ such that $\varphi_{e^{\prime}}^{e}\left(y_{e}\right)=0$. Let $\pi_{e}: F_{I} \rightarrow M_{e}$ and hence $y_{e} \pi(f)$ for some $f \in F$. But $\Theta_{e}\left(y_{e}\right)=0$ and hence $f \in K$. The desired $e^{\prime}=\left(I, S+K^{\prime}\right)$, where $K^{\prime}$ is the $\mathcal{O}_{\mathfrak{F}} G$-submodule of $K$ generated by the $\mathfrak{F}$-set $\{f\}$.

So far we have shown, that every right $\mathcal{O}_{\mathfrak{F}} G$-module is the directed colimit of finitely presented ones.

To complete the proof we need only show that there is a cofinal subset $\tilde{E}$ of $E$ such that $F_{J} / L$ is a (finitely generated) free right $\mathcal{O}_{\mathfrak{F}} G$-module for all $(J, L) \in \tilde{E}$. Let 
$(I, S) \in E$ be arbitrarily chosen. By (ii) there exists a finitely generated free right $\mathcal{O}_{\mathfrak{F}} G$-module $F$ such that the canonical map $F_{I} / S \rightarrow M$ factors through $\mathrm{F}$. We shall show that $F=F_{J} / L$ for some $(J, L) \in \tilde{E}$.

Let $B$ be a finite $\mathfrak{F}$-set, which is a basis for $F$. We can find an $\mathfrak{F}$-set $B^{\prime} \subset M \times N$ such that $B^{\prime} \cap I=\varnothing, B^{\prime}$ and $B^{\prime}$ have the same order as $\mathfrak{F}$-sets and $\pi(B)=\pi\left(B^{\prime}\right)$. We put $J=B^{\prime} \cup I$ and there is a natural surjection $\sigma: F_{J} \rightarrow K$ where $L=\operatorname{ker}(\sigma)$ is a finitely generated $\mathcal{O}_{\mathfrak{F}} G$-submodule of $K$. Thus, for each $e \in E$ there exists an $e^{\prime} \in \tilde{E}$ and an arrow $e \rightarrow e^{\prime}$, which proves our claim.

(iii) $\Rightarrow$ (i): Direct colimits commute with tensor-products over $\mathcal{O}_{\mathfrak{F}} G$, which follows from (4) and the fact that coproducts commute with $\otimes_{\mathfrak{F}}$. Also, projectives are flat.

It follows from the proof that every $\mathcal{O}_{\mathfrak{F}} G$-module is the filtered colimit of finitely presented modules. We thus obtain the following:

Corollary 3.3. Every finitely presented flat right $\mathcal{O}_{\mathfrak{F}} G$-module is projective.

Lemma 3.4. Let $\left\{P_{i}\right\}_{i \in I}$ be a countable direct system of projectives in $\operatorname{Mod}_{\mathfrak{F}}-G$. Then $\underline{\text { colim }} P_{i}=P$ has projective dimension $\leqslant 1$.

Proof. Since $I$ is countable, we can pick a cofinal System $C \subset I$, so that $P_{c_{1}} \rightarrow$ $P_{c_{2}} \rightarrow \ldots$ with $\underline{\operatorname{colim}} P_{c_{j}}=P$. Hence, the map in the explicit description of the colimit above is injective and we have a projective resolution

$$
\coprod_{\varphi: i \rightarrow j} P_{i} \hookrightarrow \coprod_{i \in C} P_{i} \rightarrow P
$$

of $P$ of length 1 .

Analogous to the finitely presented case we say a right $\mathcal{O}_{\mathfrak{F}} G$-module $M$ is countably presented if there is a short exact sequence $N \hookrightarrow F \rightarrow M$ with $F$ countably generated free and $N$ countably generated, i.e. there is a countably generated free mapping onto $N$.

Proposition 3.5. Every countably presented flat module $M \in \operatorname{Mod}_{\mathfrak{F}}-G$ has $p d_{\mathfrak{F}} M \leqslant$ 1 .

Proof. There exists an exact sequence of right $\mathcal{O}_{\mathfrak{F}} G$-modules

$$
F_{1} \stackrel{d}{\longrightarrow} F_{0} \stackrel{\pi}{\longrightarrow} M \rightarrow 0
$$

where $F_{0}$ and $F_{1}$ are countably generated free, i.e. there exist countable subfamilies $\mathfrak{F}_{0}=\left\{K_{1}, K_{2}, \ldots\right\}$ and $\mathfrak{F}_{1}=\left\{L_{1}, L_{2}, \ldots\right\}$ of $\mathfrak{F}$ such that

$$
F_{1}=\coprod_{L \in \widetilde{F}_{1}} P_{L} \quad \text { and } \quad F_{0}=\coprod_{K \in \mathfrak{F}_{0}} P_{K} .
$$

By Theorem 3.2, $M$ is the directed colimit colim $F_{\lambda}(\lambda \in \Lambda)$ of finitely generated free modules $F_{\lambda}$. Let $\mu_{\lambda}: F_{\lambda} \rightarrow M$ denote the canonical maps. Hence there exist maps $f_{\lambda}: F_{\lambda} \rightarrow F_{0}$ such that $\pi f_{\lambda}=\mu_{\lambda}$ for all $\lambda \in \Lambda$. 
For arbitrary $j \geqslant 1$ we denote by

$$
m_{j}=\max \left\{i \mid P_{K_{i}} \subseteq d\left(P_{L_{j}^{\prime}}\right) \text { for some } j^{\prime} \leqslant j\right\} .
$$

Furthermore, for each $j>0$ there exists a $\lambda(j) \in \Lambda$ such that for each $i \leqslant m_{j}$ there is a $P_{\bar{K}_{i}}$, a direct summand of $F_{\lambda(j)}$, with $f_{\lambda(j)}\left(P_{\bar{K}_{i}}\right)=P_{K_{i}}$. Therefore, for each coproduct we have $\pi\left(\coprod P_{\bar{K}_{i}}\right)=0$, i.e. $P_{\bar{K}_{i}} \subseteq d\left(F_{1}\right)$.

Thus $M$ is a direct summand of $\operatorname{colim}_{\lambda(j)}$, a countable colimit of free modules and has projective dimension 1 by Lemma 3.4.

\section{Homological and cohomological dimensions}

From now on let $\mathfrak{F}$ denote the family of all finite subgroups of a group $G$. Analogous to ordinary homology and cohomology we define (co)homological dimensions via resolutions of the trivial module $\underline{\mathbb{Z}}$, which denotes the constant functor $\underline{\mathbb{Z}}(G / K)=\mathbb{Z}$ for all $K \in \mathfrak{F}$, where all maps $\underline{\mathbb{Z}}(G / K) \rightarrow \underline{\mathbb{Z}}(G / L)$ are the identity transformation. The obvious definitions are now $\underline{\operatorname{cd}} G=p d_{\mathfrak{F}} \underline{\mathbb{Z}}$ and $\underline{\mathrm{hd}} G=f l d_{\mathfrak{F}} \underline{\mathbb{Z}}$. We shall compare the two quantities and also make some remarks about their properties.

Similiarly to ordinary group-homology, a standard resolution plays an important rôle in Bredon-Homology. We begin this section with a description of this resolution.

Let $\Delta$ denote the $\mathfrak{F}$-set with $\Delta_{K}=\{G / K\}$ for all $K \in \mathfrak{F}$. As noted in (1) the free module on $\Delta$ is of the form $F_{\Delta}=\coprod_{K \in \mathfrak{F}} P_{K}(-)$. Simultaneoulsy we shall further consider the $G$-set $\tilde{\Delta}=\coprod_{K \in \mathfrak{F}} G / K$. This set has the following property:

$$
\tilde{\Delta}^{H} \neq \varnothing \quad \Longleftrightarrow H \in \mathfrak{F} .
$$

When evaluating $F_{\Delta}$ at $G / H \in \mathcal{O}_{\mathfrak{F}} G$, we obtain the following module for the Weyl-group $W H=N_{G}(H) / H$ :

$$
F_{\Delta}(G / H)=\coprod_{K \in \mathfrak{F}} P_{K}(G / H)=\bigoplus_{K \in \mathfrak{F}} \mathbb{Z}[G / K]^{H}=\mathbb{Z} \tilde{\Delta}^{H} .
$$

Consider free $\mathcal{O}_{\mathfrak{F}} G$-modules on cartesian products of $\mathfrak{F}$-sets:

$$
\begin{aligned}
F_{\Delta \times \Delta} & =\coprod_{\left(\delta, \delta^{\prime}\right) \in \Delta \times \Delta} \mathbb{Z} \operatorname{mor}\left(-, \varphi\left(\delta, \delta^{\prime}\right)\right) \\
& =\coprod_{K, L \in \mathfrak{F}} \mathbb{Z}[\operatorname{mor}(-, G / K) \times \operatorname{mor}(-, G / L)],
\end{aligned}
$$

by (1) and hence, after evaluating at $G / H \in \mathcal{O}_{\mathfrak{F}} G$ :

$$
F_{\Delta \times \Delta}(G / H)=\bigoplus_{K, L \in \mathfrak{F}} \mathbb{Z}\left[(G / K)^{H} \times(G / L)^{H}\right]=\mathbb{Z}\left(\tilde{\Delta}^{H} \times \tilde{\Delta}^{H}\right) .
$$

We now define a map of $\mathfrak{F}$-sets

$$
d_{n}: \Delta^{(n+1)}=\underbrace{\Delta \times \ldots \times \Delta}_{n \text { times }} \rightarrow \underbrace{\Delta \times \ldots \times \Delta}_{n-1 \text { times }}=\Delta^{(n)},
$$

as follows: for each $K \in \mathfrak{F}$ put

$d_{n}\left(g_{0} K, \ldots, g_{i} K, \ldots, g_{n} K\right)=\sum_{i=0}^{n}(-1)^{i}\left(g_{0} K, \ldots, \widehat{g_{i} K}, \ldots, g_{n} K\right)$ for each $0 \leqslant i \leqslant n$, 
where $\widehat{g_{i} K}$ means leaving this term out. This now gives us a complex of free $\mathcal{O}_{\mathfrak{F}} G$ modules

$$
\ldots \rightarrow F_{\Delta^{(n+1)}} \rightarrow F_{\Delta^{(n)}} \rightarrow \ldots \rightarrow F_{\Delta} \stackrel{\varepsilon}{\longrightarrow} \underline{\mathbb{Z}} \rightarrow 0 .
$$

The map $\varepsilon: F_{\Delta} \rightarrow \underline{\mathbb{Z}}$ is defined by $\varepsilon(g K)=1$.

When evaluating (10) at $G / H \in \mathcal{O}_{\mathfrak{F}} G$, we obtain a sequence of $W H$-modules

$$
\rightarrow \mathbb{Z}\left(\tilde{\Delta}^{H}\right)^{(n+1)} \rightarrow \mathbb{Z}\left(\tilde{\Delta}^{H}\right)^{(n)} \rightarrow \ldots \rightarrow \mathbb{Z} \tilde{\Delta}^{H} \rightarrow \mathbb{Z} \rightarrow 0,
$$

where the induced connecting maps are as follows: $\tilde{d}^{n}\left(\delta_{0}, \ldots, \delta_{i}, \ldots, \delta_{n}\right)=$ $\sum_{i=0}^{n}(-1)^{i}\left(\delta_{0}, \ldots, \hat{\delta}_{i}, \ldots, \delta_{n}\right)$, where the $\delta_{i} \in \tilde{\Delta}^{H}$ for all $0 \leqslant i \leqslant n$. It was shown in [19, Lemma 3.1], that the $G$ sets $\tilde{\Delta}^{H}$ satisfy condition (7) and furthermore that the sequence (11) is an exact sequence of $W H$-modules and hence the standard resolution (10) is indeed an exact sequence of right $\mathcal{O}_{\mathfrak{F}} G$-modules.

Theorem 4.1. Let $G$ be an arbitrary group. Then

(i) $\underline{\operatorname{hd}} G \leqslant \underline{\mathrm{cd}} G$

(ii) When $G$ is countable then $\underline{\mathrm{cd}} G \leqslant \underline{\mathrm{hd}} G+1$

Proof. (i) follows directly from the fact that projectives are flat. Being countable, $G$ has only countably many finite subgroups. Hence the standard resolution (10) is a countable free resolution and (ii) follows from Proposition 3.5.

Following [16] we say a family $\mathfrak{F}$ is bounded if each $K \in \mathfrak{F}$ is contained in a maximal $M \in \mathfrak{F}$.

Theorem 4.2. Let $\left\{G_{\lambda}\right\}_{\lambda \in \Lambda}$ be a direct system of groups. Then, for the direct limit $G=\lim _{\rightarrow} G_{\lambda}$ and $\mathfrak{F}$ a bounded family of finite subgroups of $G$,

(i) $\underline{\mathrm{hd}} G=\max \left\{\underline{\mathrm{hd}} G_{\lambda}\right\}$

(ii) If $\Lambda$ is countable then $\underline{\operatorname{cd}} G \leqslant \max \left\{\underline{\operatorname{cd}} G_{\lambda}\right\}+1$

Proof. Let $\iota_{\lambda}: G_{\lambda} \rightarrow G$ and $\varphi_{\mu}^{\lambda}: G_{\lambda} \rightarrow G_{\mu}$ be the canonical maps for all $\lambda, \mu \in \Lambda$. Let $\Delta_{\lambda}$ denote the $\operatorname{Obj}\left\{\mathcal{O}_{\mathfrak{F}} G_{\lambda}\right\}$-set with $\Delta_{\lambda, K_{\lambda}}=\left\{G_{\lambda} / K_{\lambda}\right\}$ for all $K_{\lambda} \in \mathfrak{F}_{\lambda}$, the family of all finite subgroups of $G_{\lambda}$. This is in a one-to-one correspondence with the $\operatorname{Obj}\left\{\mathcal{O}_{\mathfrak{F}} G\right\}$-set (or $\mathfrak{F}$-set) for short) $\Delta_{\lambda}^{\prime}$, which is defined as $\Delta_{\lambda, K}=\{G / K\}$ for all $K \in \mathfrak{F}$ with $K=\iota_{\lambda}\left(K_{\lambda}\right)$ and empty otherwise. By some abuse of notation we denote both with $\Delta_{\lambda}$.

We have a directed system of $\mathfrak{F}$-sets given through:

$$
\begin{array}{ccc}
\Delta_{\lambda} & \rightarrow & \Delta_{\mu} \\
G_{\lambda} / K_{\lambda} & \mapsto & G_{\mu} / K_{\mu}
\end{array}
$$

for all $\varphi_{\mu}^{\lambda}: G_{\lambda} \rightarrow G_{\mu}$. Since $\mathfrak{F}$ is bounded, $\underline{\operatorname{colim}} \Delta_{\lambda}=\Delta$, which is the $\mathfrak{F}$-set defined by $\Delta_{K}=\{G / K\}$ for all $K \in \mathfrak{F}$.

It now follows that

$$
F_{\Delta(n)} \cong F_{\operatorname{colim} \Delta_{\lambda}^{(n)}} \cong \underline{\operatorname{colim}}_{\longrightarrow} F_{\Delta_{\lambda}^{(n)}}
$$


for all $n \geqslant 0$. Since colimits are exact (3.1) we have that $\operatorname{ker}\left(F_{\Delta^{(n)}} \rightarrow F_{\Delta^{(n-1)}}\right)=$ $K_{n} \cong \underset{\operatorname{colim}}{\longrightarrow} K_{n, \lambda}$, the $\mathrm{n}$-th kernel in the respective standard resolutions for $\mathcal{O}_{\mathfrak{F}} G_{\lambda}$. (i) now follows directly and (ii) from Lemma 3.4.

Proposition 4.3. For every group $G, \underline{\mathrm{hd}} G \geqslant \operatorname{hd}_{\mathbb{Q}} G$.

Proof. Let $\underline{\mathrm{hd}} G=n$. Take an arbitrary free resolution of $\underline{\mathbb{Z}}$. Hence the n-th kernel is flat and by 3.2 a direct limit of free modules. Evaluating at $G / 1$ and tensoring the result with $\mathbb{Q}$ gives us a $\mathbb{Q} G$-flat resolution of $\mathbb{Q}$, where the n-th kernel is a direct limit of free modules, hence flat.

\section{Extensions}

In this section we discuss the Bredon homological and cohomological dimensions for extensions $N \hookrightarrow G \rightarrow Q$ of groups. Our results are applications of Martinez' spectral sequences $[\mathbf{1 6}]$. We begin by recalling her results and adapting them to our setting.

Let us denote by $\mathfrak{F}(G), \mathfrak{F}(N)$ and $\mathfrak{F}(Q)$ the families of finite subgroups of $G, H$ and $Q$ respectively.

Let $\mathfrak{H}=\{S \leqslant G: N \leqslant S$ and $S / N \in \mathfrak{F}(Q)\}$. We also have a map

$$
\begin{aligned}
\pi: \mathfrak{F}(G) & \rightarrow \mathfrak{H} \\
L & \mapsto L N
\end{aligned}
$$

and there is an obvious equivalence of categories between $\operatorname{Mod}_{\mathfrak{F}(Q)}-Q$ and $\operatorname{Mod}_{\mathfrak{H}}-G$ and between $Q-\operatorname{Mod}_{\mathfrak{F}(Q)}$ and $G-\operatorname{Mod}_{\mathfrak{H}}$. This implies in particular that

$$
c d_{\mathfrak{H}} G=\underline{\mathrm{cd}} Q \quad \text { and } \quad h d_{\mathfrak{H}} G=\underline{\mathrm{hd}} Q .
$$

An application of Martinez' spectral sequence $[\mathbf{1 6}, 5.1]$ now yields the following result:

Theorem 5.1. [16, 5.2] Let $N \hookrightarrow G \rightarrow Q$ be an extension of groups. Assume that there are integers $m$ and $n$ such that for every $S \in \mathfrak{H}, \underline{\mathrm{cd}} S \leqslant n$ and $\underline{\mathrm{hd}} S \leqslant m$. Then

$$
\underline{\mathrm{cd}} G \leqslant m+\underline{\mathrm{cd}} Q
$$

and

$$
\underline{\mathrm{hd}} G \leqslant m+\underline{\mathrm{hd}} Q .
$$

Example 5.2. The conditions of theorem 5.1 are obviously satisfied when $Q$ is torsion-free. Here the theorem says that $\underline{\mathrm{cd}} G \leqslant \underline{\mathrm{cd}} N+\operatorname{cd} Q$ and $\underline{\mathrm{hd}} G \leqslant \underline{\mathrm{hd}} N+\mathrm{hd} Q$.

Example 5.3. Theorem 5.1 can also be applied when $N$ is polycyclic. See for example [15, Example 5.25], where it is shown that for a polycyclic-by-finite group there is always a model for an $\underline{E} G$ of dimension equal to the Hirsch-rank if its polycyclic subgroups of finite index. Hence, for all $S \in \mathfrak{H}, \underline{\mathrm{cd}} S=\underline{\mathrm{hd}} S=h N$.

Example 5.4. We cannot hope to get a result like 5.1 for arbitrary group-extensions. Ian Leary and the author give examples of virtually torsion-free groups $G$, where 
$\underline{\mathrm{cd}} G$ is strictly greater than $\mathrm{cd} H$ for $H$ a torsion-free subgroup of finite index, see [12, Example 12]. Since $H$ is of type $F P_{\infty}$, the homological and cohomological dimensions are equal [1, Theorem 4.6]. Analogous to the proof of [12, Theorem 6] we can use the homology of $\underline{E} G$ relative to its singular set to provide a lower bound for hd $G$. Let $N$ be a $\mathbb{Z} G$-module and let $I_{1} N$ be the covariant $\mathcal{O}_{\mathfrak{F}} G$-module defined as $I_{1} N(G / K)=0$ for all $K \neq\{1\}$ and $I_{1} N(G / 1)=N$. It can be checked that $H_{*}^{G}\left(\underline{E} G, \underline{E} G^{\text {sing }} ; N\right) \cong \operatorname{Tor}_{*}^{\mathfrak{F}}\left(\underline{\mathbb{Z}}, I_{1} N\right)$. For the above examples there is a model $X$ for $\underline{E} G$ such that the relative cellular chain complex $C_{*}\left(X, X^{\text {sing }}\right)$ contains as a direct summand a chain complex consisting of only one copy of $\mathbb{Z} G$ in the top degree $n=\underline{\operatorname{gd}} G$. Hence for our examples $H_{n}^{G}\left(\underline{E} G, \underline{E} G^{\text {sing }} ; \mathbb{Z} G\right) \neq 0$ and therefore

$$
\underline{\mathrm{hd}} G=\underline{\mathrm{cd}} G>\operatorname{cd} H=\operatorname{hd} H .
$$

In the next section we take a closer look at nilpotent groups where we can prove some more detailed results. Note that Theorem 5.1 can be applied to the case when $N$ is finite. But an application of Martinez' spectral sequence [16, 4.3] yields the follwong stronger result.

Theorem 5.5. Let $F \hookrightarrow G \rightarrow Q$ be an extension of groups where $F$ is finite. Then

$$
\underline{\mathrm{cd}} G=\underline{\mathrm{cd}} Q \quad \text { and } \underline{\mathrm{hd}} G=\underline{\mathrm{hd}} Q .
$$

Proof. Since $F$ is finite, $\mathfrak{H} \subseteq \mathfrak{F}(G)$ and also, for each $L \in \mathfrak{F}(G)$, there is $S=L F \in \mathfrak{H}$ such that $L \leqslant S$. Hence the conditions of $[\mathbf{1 6}, 4.5]$ apply and $H_{\mathfrak{H}}^{n}(G, C) \cong H_{\mathfrak{F}}^{n}(G, C)$ and $H_{n}^{\mathfrak{H}}(G, D) \cong H_{n}^{\mathfrak{F}}(G, D)$ for all $C \in \operatorname{Mod}_{\mathfrak{F}^{-}} G$ and all $D \in G$-Mod $\operatorname{Mod}_{\mathfrak{F}}$.

\section{Nilpotent groups}

In this section we consider nilpotent groups $G$. The elements of finite order in $G$ form a charcteristic locally finite subgroup, denoted $\tau(G)$. We write $h G$ for the Hirsch length of $G$.

Theorem 6.1. Let $G$ be a nilpotent group and $\mathfrak{F}$ be the family of finite subgroups of $G$. Then

$$
\underline{\mathrm{hd}} G=h G .
$$

Proof. Obviously, from 4.3 and Stammbach's result [23] it follows that $\underline{\mathrm{hd}} G \geqslant h G$. We need to show the other inequality.

Let $G$ be of finite Hirsch length $n$. We have a group extension

$$
\tau(G) \hookrightarrow G \rightarrow H
$$

with $H$ torsion-free, see e.g. [20], page 132. Since $H$ is torsion-free, Stammbach's result $[\mathbf{2 3}]$ implies that $h H=\mathrm{hd} H=\underline{\mathrm{hd}} H$.

We now use a similar argument as in Theorem 5.5, but this time we apply it to $\hat{\mathfrak{F}}$, the completion of $\mathfrak{F}$. Now $\mathfrak{H}=\{\tau(G)\}$, and the families $\mathfrak{H}$ and $\hat{\mathfrak{F}}$ satisfy the conditions of $[\mathbf{1 6}, 4.5]$ and hence $\operatorname{hd}_{\mathfrak{H}} G=\operatorname{hd}_{\widehat{F}} G$. Also, Theorem 4.8 of $[\mathbf{1 6}]$ implies that $h d_{\widehat{\mathfrak{F}}} G \geqslant \underline{\mathrm{hd}} G$. As the Hirsch length is additive for group-extensions we obtain the following chain of inequalities:

$$
h G=h H=\underline{\mathrm{hd}} H=\operatorname{hd}_{\mathfrak{H}} G=\operatorname{hd}_{\widehat{\mathfrak{F}}} G \geqslant \underline{\mathrm{hd}} G \geqslant h G,
$$


which proves the theorem.

Remark 6.2. Theorem 4.1 gives us bounds for the Bredon cohomological dimension of nilpotent groups. For virtually torsion-free nilpotent groups the next result is of interest, linking $\underline{\mathrm{cd}} G$ to the virtual cohomological dimension $(v c d G)$ of $G$.

Theorem 6.3. Let $G$ be a virtually torsion-free nilpotent group. Then $\underline{\mathrm{cd}} G=v c d G$.

Proof. Let $N \hookrightarrow G \rightarrow Q$ be an extension with $N$ torsion-free and $Q$ finite. As $N$ is torsion-free, the intersection $\tau(G) \cap N$ is trivial. Therefore $\tau(G)$ is isomorphic to a subgroup of $Q$ and hence finite. Now apply Theorem 5.5 to the extension $\tau(G) \hookrightarrow G \rightarrow H$ and use the fact that $N$ is isomorphic to a subgroup of finite index in $H$.

Remark 6.4. This theorem can be used to verify that the Brown conjecture holds for arbitrary virtually torsion-free nilpotent groups. It follows directly for groups of $\underline{\mathrm{cd}} G \neq 2$ from the results of Dunwoody [7] and Lück [13], as here we can always find a model for $\underline{E} G$ of dimension equal to $\underline{\mathrm{cd}} G$. We only have to make sure that an Eilenberg-Ganea type phenomenon cannot happen for virtually torsion-free nilpotent groups of $\underline{\operatorname{cd}} G=2$. Let $N$ be a torsion-free subgroup of finite index in $G$. Then $\operatorname{cd} N=2$. Applying [1, Theorem 8.8] to $N$ and using that the centre $Z(N) \neq\{1\}$ yields $\operatorname{cd} Z(N)=1$. Hence $Z(N)$ is infinite cyclic. The extension $Z(N) \hookrightarrow N \rightarrow Q$ now satisfies the assumptions of Fel'dman's theorem, see Theorem 5.5 and Remark of $[\mathbf{1}]$. Hence $2=\operatorname{cd} N=\operatorname{cd} Z(N)+\operatorname{cd} Q$ and $\operatorname{cd} Q=1$ implying that $Q$ is free abelian of rank 1. Hence $N$ is polycyclic and therefore admits a 2-dimensional model for an Eilenberg-Mac Lane space $E G$. Applying this to $\tau(G) \hookrightarrow G \rightarrow H$ implies that $G$ is an extension of finitely generated groups, hence finitely generated and in particular polycylic. And for a polycyclic group of $\underline{\operatorname{cd}} G=2$ we can always find a 2-dimensional model for $\underline{E} G$, see [15, Example 5.25].

\section{Acknowledgements}

The author thanks Conchita Martinez-Pérez for many interesting and fruitful discussions and also for making early versions of $[\mathbf{1 6}]$ available to her. Thank you also to Ian Leary for making useful remarks on earlier versions, and to the referee of an earlier version, whose comments led to improvements to Section 6 and the inclusion of Section 5 .

\section{References}

[1] R. Bieri, Homological dimension of discrete groups, Queen Mary College Mathematics Notes, London (1976).

[2] N.P. Brady, I.J. Leary and B.E.A. Nucinkis On algebraic and geometric dimensions for groups with torsion, J. London Math. Soc. 64 (2) (2001) 489500 .

[3] G.E. Bredon Equivariant cohomology theories, Springer Lecture Notes in Math. 341967. 
[4] K.S. Brown Groups of virtually finite dimension, in Homological Group Theory, London Math. Soc. Lecture Notes 36 Cambridge Univ. Press (1979), $27-70$.

[5] K.S. Brown Cohomology of groups Graduate Texts in Mathematics 87, Springer Verlag (1982).

[6] T. tom Dieck, Transformation Groups, de Gruyter Studies in Mathematics 8 (1987).

[7] M.J. Dunwoody, Accessibility and groups of cohomological dimension 1, Proc. London Math. Soc. 38 (1997), 193-215.

[8] S. Eilenberg and T. Ganea, On the Lusternik-Schnirelmann category of abstract groups, Ann. of Math. 65 (1957), 517-518.

[9] J.L. Fisher, The Tensor Product of Functors; Satellites; and Derived Functors, J. Algebra 8 (1968) 277-294.

[10] K.W. Gruenberg, Cohomological topics in group theory, Lecture Notes in Mathematics 142 (Springer, Berlin, 1970).

[11] D. Lazard, Autour de la platitude, Bull. Soc. math. France 97 (1969), 81-128.

[12] I.J. Leary and B.E.A. Nucinkis, Some groups of type VF, Invent. Math. 151 (1) (2003), 135-165.

[13] W. Lück, Transformation groups and algebraic K-theory, Lecture Notes in Mathematics 1408 (Springer, Berlin 1989).

[14] W. Lück, The type of the classifying space for a family of subgroups, J. Pure Appl. Algebra 149 (2000), 177-203.

[15] W. Lück, Survey on Classifying Spaces for Families of Subgroups, preprint (2003).

[16] C. Martinez-Pérez, A Spectral sequence in Bredon (co)homology, J. Pure Appl. Algebra 176 (2002) 161-173.

[17] J.P. May Equivariant Homotopy and Cohomology Theory, AMS Regional Conference Series in Mathematics 91, (1996).

[18] G. Mislin, Equivariant K-homology of the classifying space for proper actions, In Notes on the Advanced Course on proper group actions. CRM Barcelona (2001).

[19] B.E.A. Nucinkis, Is there an easy algebraic characterisation of universal proper $G$-spaces?, manuscripta math. 102 (2000) 335-345.

[20] D.J.S. Robinson, A Course in the Theory of Groups, 2nd ed. Graduate Texts in Mathematics 80, Springer Verlag (1995).

[21] D. Segal, Polycyclic groups, Cambridge University Press (1983). 
[22] J. Slominska, Hecke structure on Bredon cohomology, Fundamenta Mathematicae 140 (1991) 1-30.

[23] U. Stammbach, On the weak homological dimension of the group algebra of solvable groups, J. London Math. Soc (2) 2, (1970), 567 - 570.

[24] C.A. Weibel, An introduction to homological algebra, Cambridge studies in advanced mathematics 38, Cambridge University Press (1994).

This article may be accessed via WWW at http://www.rmi.acnet.ge/hha/ or by anonymous ftp at

ftp://ftp.rmi.acnet.ge/pub/hha/volumes/2004/n1a4/v6n1a4.(dvi,ps,pdf)

Brita E. A. Nucinkis bean@math.ethz.ch

Departement Mathematik,

ETH-Zentrum,

CH 8092 Zürich 